\title{
328 - Depression as a disorder of accelerated ageing
}

\section{Presenting author:}

- Prof.dr. Richard C. Oude Voshaar, University of Groningen, The Netherlands (r.c.oude.voshaar@umcg.nl)

\section{Co-author:}

- Prof.dr. Ivan Aprahamian, Faculty of Medicine of Jundiaí, Brazil (ivan.aprahamian@gmail.com)

Depressive disorder has been conceptualised as a disorder of accelerated ageing. Furthermore, metaanalyses have shown that depression associated with excess mortality, although most studies can be criticised for insufficient confounder control. These characteristics of depression resemble the characteristics of biomedical frailty. Biomedical frailty can be assessed with the frailty index (FI) based on the deficit accumulation model. This model postulates that the proportion of at least 30 ageingrelated health deficits reflects biological age on top of chronological age. The characteristics of the $\mathrm{Fl}$ are independent of the specific health deficits included, as long as health deficits from different physiological systems are included. The Fl is the most accurate frailty model for predicting mortality, the ultimate outcome of increasing frailty states. Because of its continuous nature, the $\mathrm{Fl}$ is sensitive to change which enables us to study trajectories of frailty.

The objective of the present lecture is 1) to show that depressive disorder associated with accelerated biological ageing as indexed by the frailty index, 2) that depressive disorder is a risk factor for excess mortality, and 3) to show that this latter explanation is largely explain by biomedical frailty.

The Netherlands Study of Depression in Older persons (NESDO) is a clinical cohort of 378 depressed patients according to DSM-IV criteria and 132 non-depressed controls who have been followed up for six-year. The data of this cohort study enabled us to construct a frailty index based on 41 nondepression related health deficits. Linear mixed models showed that the frailty index had a significantly steeper increase over six years among depressed patients compared to their non-depressed counterparts.

Subsequently, we constructed a frailty index based on 64 health deficits in the Lifelines cohort study, a three-generation population-based study including 167,000 persons. In this population-based cohort, we replicated our finding that the presence of a depressive disorder according to DSM-IV criteria, but also anxiety disorders, were associated with an accelerated increase of the frailty index over a 5-year follow-up. Furthermore, Cox-regression showed that the presence of a depressive disorder or any anxiety disorder was also associated with excess mortality over a 10-year follow-up. These effects remained significant when adjusted for socio-demographic characteristics, lifestyle variables and multimorbidity, but disappeared when adjusted for the frailty index.

We conclude that depressive disorder can indeed be conceptualised as a disorder of accelerated biological ageing, as indexed by the frailty index. Moreover, biomedical frailty may be an explanatory factor for excess mortality found to be associated with affective disorders. Therefore, biomedical frailty seems a promising marker for risk stratification in geriatric psychiatry. 